\section{Atmospheric Light Columns from Artificial Lights.}

BrILLIANT beams of light, appearing to go upwards vertically from street lamps and electric signs, are seen here frequently during the winter months. The accompanying photograph (Fig. 1), taken on the evening of $\mathrm{Feb} .4$, shows a typical case.

The beams are seen only when the air is filled with snow or ice crystals. These apparently act as reflectors, since the beams from neon signs show the same colour red as the source itself. Examination of the beams through a Nicol prism indicates that they are unpolarised.

An exceptional case was observed on the evening of Jan. 3. A powerful electric sign showed four beams in addition to the vertical beam. Two of

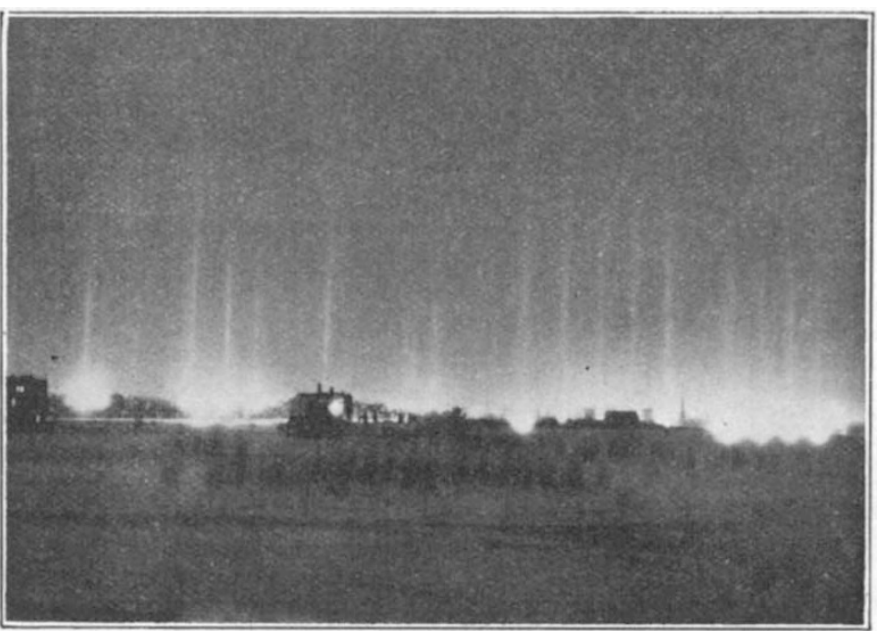

FIG. 1. horizontal surface at a height of 500 feet would serve to produce rays coming down at that angle. The narrowness of the beams is in favour of this view of the phenomenon.

As to the exceptional observation of Jan. 3, it is likely that crystals of two types were present ; laminar crystals were responsible for the vertical column. On the analogy of the mock-sun ring, the horizontal beams must have been due to the reflection of light from the faces of prisms floating with their axes vertical. Two reasons for the assumption of this position are known. Either the crystals contain air-bubbles or they have flat crystals attached to them to serve as parachutes. The latter explanation is the more likely in the present instance, for flat crystals were certainly present, and it is known that prisms can grow on the under sides of flat crystals.

The beams inclined at $45^{\circ}$ have no analogue in daylight observations. Such beams might be due to light reflected twice, once at a vertical surface and once at a horizontal surface.

The growth of brilliant illumination in the cities of the north will provide an opportunity for the observation of various other phenomena. If an artificial mocksun ring can be seen, the mock-suns them. selves should be looked for as well as the circular halo. Mr. Currie is to be congratulated on his pioneer work in this field. The suggestion may be added that when possible the forms of the crystals should be recorded as well as the optical phenomena attributed to their presence.

Kew Observatory

$$
\text { F. J. W. WHIPPLE. }
$$

Feb. 26.

\section{The Heat of Dissociation of Oxygen and of the $\mathrm{C}-\mathrm{H}$ Bond.}

Is a short notice in Die Naturwissen-

these were horizontal, while the other two were in clined at an angle of about $45^{\circ}$ with the horizontal. The horizontal beams were apparently of the same intensity as the vertical beam. The inclined beams were just visible.

Up to the present $I$ have been unable to find a mention of this phenomena in the literature on the subject, or to suggest a reasonable explanation.

University of Saskatchewan,

Saskatoon, Sask., Feb. 8.

Lighт columns over artificial lights have been described previously, but $\mathrm{Mr}$. Currie appears to be the first to photograph the phenomenon.

The columns can be explained in the same way as sun pillars. Mr. Currie suggests that the light is reflected from the surfaces of ice crystals. We may add that to produce vertical columns the reflecting surfaces must be nearly horizontal. The effective crystals are probably laminar. A flat disc falling slowly through the air keeps nearly horizontal.

In the explanation of the sun pillar, stress has to be laid on the fact that the falling disc will wobble or even spin so that the reflecting surfaces are not strictly horizontal and the pillar is analogous to the streak of light produced by reflection from rippled water. Columns such as are shown in Fig. 1 might be due, however, to reflection from strictly horizontal surfaces at different heights in the atmosphere. The elevation of the tallest of the columns may be estimated at about $12^{\circ}$. If the source of this light was a mile from the camera, reflection from a schaften, Dec. 20, 1929, I published a new method of determining the heat of dissociation of oxygen (128 kcal.), comparing the spectroscopical dissociation of nitrogen peroxide with the thermal decomposition according to the scheme

$$
\begin{array}{ll}
\text { Photochemical : } & \mathrm{NO}_{2}=\mathrm{NO}+\mathrm{O}-77 \mathrm{kcal} .(\mathrm{A3700}) \\
\text { Thermal: } & \mathrm{NO}_{2}=\mathrm{NO}+\frac{1}{2} \mathrm{O}_{2}-13 \mathrm{kcal} .
\end{array}
$$

In a letter in Nature of Feb. 8, p. 202, Prof. V. Henri has calculated-using exactly the same method and values adopted by me in my note-this heat of dissociation, and arrives, of course, at exactly the same value of $128 \mathrm{kcal}$. It should be stated here that my computation of the dissociation energy was based not only on the fine work of Norrish on the photochemical decomposition of nitrogen peroxide but also on my own spectroscopical investigations of the absorption spectrum of this molecule. I found that predissociation took place between $\lambda 3700$ and $\lambda 3800 \mathrm{~A}$. A full account of this new method of determining spectroscopically heats of dissociation was given at the meeting of the Physikalische Gesellschaft at Kreuznach on Nov. 23, 1929, and will be published in Zeits. f. phys. Chemie (B).

I would especially wish to emphasise here that my method may readily be extended to other problems. From the thermal decomposition of ozone according to the analogous scheme

$$
\begin{array}{ll}
\text { Photochemical : } & \mathrm{O}_{3}=\mathrm{O}_{2}+\mathrm{O}-30 \mathrm{kcal} .(\lambda 9500) \\
\text { Thermal : } & \mathrm{O}_{3}=\mathrm{O}_{2}+\frac{1}{2} \mathrm{O}_{2}+34 \mathrm{kcal} .
\end{array}
$$

it follows that photochemical decomposition should start at about $\lambda 9500$ if the dissociation products are normal $\mathrm{O}_{2}$ and normal $\mathrm{O}$, or at about $\lambda 4100$ or less, if some excited molecule or atom is the end product. 